\title{
LOCAL STEREOLOGY OF EXTREMES
}

\author{
ZBYNĚK PAWLAS ${ }^{\bowtie}$ \\ Department of Probability and Mathematical Statistics, Faculty of Mathematics and Physics, Charles University, \\ Sokolovská 83, 18675 Prague, Czech Republic \\ e-mail: pawlas@karlin.mff.cuni.cz \\ (Received November 28, 2011; revised April 2, 2012; accepted April 4, 2012)
}

\begin{abstract}
Local stereology uses information obtained from central sections passing through a reference point of the particle. The aim of this paper is to investigate the prediction of extremes of shape and size parameters based on the central sections. We consider the particle population formed by spheroids (either prolate or oblate) and assume that the reference point is the centre of the spheroid. A relation between shape and size parameters of the particles and their planar sections is derived and consequently stability properties of the domain of attractions are proved.
\end{abstract}

Keywords: extremes, local stereology, maximum domain of attraction, shape and size parameters, spheroids.

\section{INTRODUCTION}

Local stereological methods form quite modern branch of stereology, see Vedel Jensen (1998) for a comprehensive exposition. They require that we associate a reference point to each particle and accomplish sectioning through this reference point. We speak about central sections or local probes. Information about the particle population is then extracted from these local probes. In contrast to traditional sampling, where the system of particles is sectioned by an isotropic uniform probe, the local sample is representative for the particle population. This fact was exploited in Pawlas et al. (2009) for the development of a statistical procedure for obtaining information about particle size distribution from central sections without specific assumptions about particle shape. The motivation for local stereology comes from the study of biological tissues. The particles are cells and the centres of cell nuclei or nucleoli serve as reference points. Local techniques are most easily implemented if optical sectioning is available.

One of the possible applications in stereology is to estimate extremes of particle parameters from the observation of test probes of lower dimension. This field is referred to as stereology of extremes (Beneš and Rataj, 2004). In practical applications it is often important to analyze extremes of the particle parameters. For example, the damage of materials is related rather to extremal than mean characteristics of microstructure. The application of stereology of extremes to metallurgy is discussed e.g., in Takahashi and Sibuya (2002) or Bortot et al. (2007). So far in the literature concerning stereology of extremes, only isotropic uniform random probes were considered. In Wicksell's corpuscle problem (Wicksell, 1925), the prediction of maximum size of spherical particles was studied by Drees and Reiss (1992) and Takahashi and Sibuya (1996; 1998; 2001) while the behaviour of minimum size was investigated in Kötzer and Molchanov (2006). An extension to spheroidal particles leads to the prediction of not only extremal size but also extremal shape. This was considered in Hlubinka (2003a;b; 2006a); Hlubinka and Kotz (2010) for oblate spheroidal particles and in Hlubinka $(2006 \mathrm{~b} ; \mathrm{c})$ for prolate spheroidal particles. The novel contribution of this paper is the study of stereology of extremes for spheroidal particles under different stereological sampling design. Our aim is to investigate the stereological estimation of the tail of particle shape and size distribution based on the local probes. We derive the relation between the particle parameters and the parameters of particle central section (also called profile). Subsequently, we show how the limiting distribution of the extremal particle parameters is related to the limiting distribution of the extremal profile parameters. It turns out that the distribution of profile parameters belongs to the same domain of attraction as the distribution of particle parameters.

Particular attention is devoted to the population of spheroids (either prolate or oblate). If we consider a spheroid with two semiaxes of length $a$ and one semiaxis of length $c$, then its planar section through the centre is an ellipse with semiaxes of lengths $a$ and $d$, where $\min (a, c) \leq d \leq \max (a, c)$. In other words, the length of one semiaxis is directly observed at the central section while the second semiaxis appears shorter (for prolate spheroid) or longer (for oblate 
spheroid). Therefore, profiles have more circular shape than original particles projected to the plane perpendicular to one of semiaxes of length $a$.

This paper is organized as follows. At first, we derive the relation between particle and profile shape and size parameters in the population of spheroidal particles. Then, we summarize basic facts from extreme value theory which will be needed to obtain main results stated in the subsequent section. We make the inference about the extremal domains of attraction under local sampling design. We conclude with an example showing the behaviour of maximal shape parameter in the population of simulated oblate spheroids.

\section{PARTICLES}

Local stereological estimates are based on information collected on a section plane through a fixed reference point of the particle. In the present paper, we will concentrate on three-dimensional particles and two-dimensional section planes. By a particle $K$ we understand a non-empty compact subset of $\mathbb{R}^{3}$. We associate a reference point $x$ to the particle $K$. A section plane through the reference point takes the form $x+L$, where $L$ is a fixed twodimensional linear subspace of $\mathbb{R}^{3}$. The information about the particle is deduced from the planar central section $K \cap(x+L)$. In this paper we pursue the modelbased approach. However, all results remain valid in the same way for the design-based approach. In the latter, the particle $K$ is deterministic while the section plane is random.

The class of all particles equipped with the Hausdorff metric forms a separable metric space. Thus, it is possible to define random particles, their distribution and independence. For more details on theory of random sets we refer to Molchanov (2005).

We consider a population $\Xi_{1}, \ldots, \Xi_{n}$ of random particles with reference points $X_{1}, \ldots, X_{n}$. The particles are not observed directly, only their planar profiles $\Xi_{i} \cap\left(X_{i}+L\right)$ are available. An illustration for twodimensional particles and one-dimensional central sections is shown in Fig. 1.

We assume that the particles $\Xi_{1}, \ldots, \Xi_{n}$ are independent and identically distributed. Denote by $\Xi_{0}$ a random particle with the same distribution as the $\Xi_{i}$. We will refer to $\Xi_{0}$ as a typical particle. Let $p$ be a real measurable function defined on the space of all particles. It will be used to describe shape or size parameters of particles. Ouraim is to investigate the

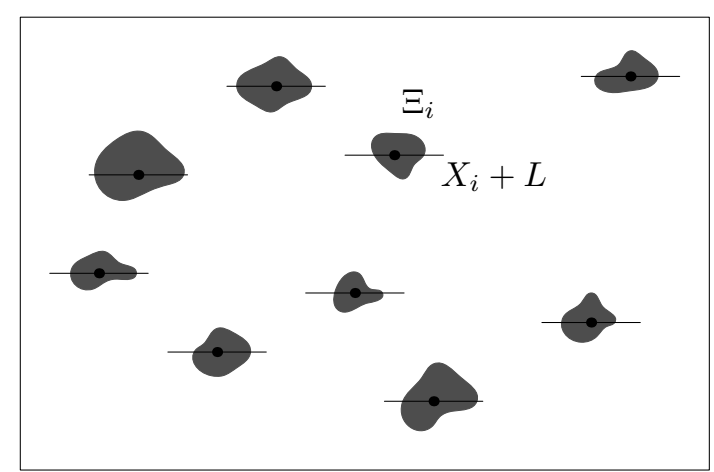

Fig. 1. A two-dimensional illustration of local sectioning applied to the particle population under study.

tail behaviour of the typical particle parameter $p\left(\Xi_{0}\right)$. Since the parameters $p\left(\Xi_{1}\right), \ldots, p\left(\Xi_{n}\right)$ describing random shape or size of particles cannot be directly observed, we have to use the particle profile parameters $q\left(\Xi_{1} \cap\left(X_{1}+L\right)\right), \ldots, q\left(\Xi_{n} \cap\left(X_{n}+L\right)\right)$ derived from local probes. In what follows, we will consider uniform randomly oriented (isotropic) particles. If the particles cannot be regarded as isotropic, we may instead randomize the orientation of the section plane (use isotropic subspace $L$ ) and work under design-based setting.

If $\Xi_{0}$ is a triaxial ellipsoid with semiaxes of lengths $A, B$ and $C(A \geq B \geq C)$ and the corresponding reference point $X_{0}$ is the centre of the ellipsoid, then the observed planar profile $\Xi_{0} \cap\left(X_{0}+L\right)$ is an ellipse. In particular, we will consider the case of two equal semiaxes, i.e., $\Xi_{0}$ is a spheroid. It can be either prolate ( $B=C$, two equal minor semiaxes) or oblate $(A=B$, two equal major semiaxes). A possible choice for $p$ describing shape of the typical particle is given by the shape factor

$$
S=\frac{A^{2}}{C^{2}}-1 .
$$

This parameter is commonly used for spheroids, see, e.g., Beneš and Rataj (2004); Hlubinka (2003a; 2006a;b). For spherical particles $(A=C)$ we get $S=0$, more elongated spheroids lead to larger value of $S$. In similar way, we can define the function $q$ to quantify the shape of the planar profile. We will denote the profile shape factor by $T$.

Each spheroid is uniquely determined by the centre $X$ (reference point), the length $A$ of major semiaxis, the length $C$ of minor semiaxis, and the angles $\Theta$ and $\Phi$ which describe its orientation. Let $\Theta$ be chosen as the angle between the norm vector of $L$ and the major (for prolate case) or minor (for oblate case) axis, i.e., $\Theta$ is the latitude and $\Phi$ is the longitude. In what follows we assume that the random vectors $(A, C)$ and $(\Theta, \Phi)$ are independent. The profile observed on planar central 
section is an ellipse and it can be easily shown that it has semiaxes of lengths

$$
D=\sqrt{\frac{A^{2} C^{2}}{C^{2} \sin ^{2} \Theta+A^{2} \cos ^{2} \Theta}}
$$

and $C$ in the case of prolate spheroid and

$$
D=\sqrt{\frac{A^{2} C^{2}}{A^{2} \sin ^{2} \Theta+C^{2} \cos ^{2} \Theta}}
$$

and $A$ for oblate spheroid. Shape factor of the ellipse obtained from central section is

$$
T=\frac{D^{2}}{C^{2}}-1=\frac{S \sin ^{2} \Theta}{1+S \cos ^{2} \Theta}
$$

for prolate spheroid and

$$
T=\frac{A^{2}}{D^{2}}-1=S \sin ^{2} \Theta
$$

for oblate spheroid. Note that $T \leq S$ in both cases. It means that the profile shape factor is always smaller or equal to the particle shape factor. Equality occurs if and only if the section plane is perpendicular to the plane spanned by two semiaxes of the same length.

Since the spheroid $\Xi_{0}$ is assumed to have isotropic orientation, the density of $\Theta$ is $f_{\Theta}(\theta)=\sin \theta, \theta \in$ $[0, \pi / 2]$. Exploiting the independence of $\Theta$ and $S$, we obtain the complementary distribution functions of $T$ :

$$
\begin{aligned}
1-F_{T}(t) & =\mathbb{P}\left(\frac{S \sin ^{2} \Theta}{1+S \cos ^{2} \Theta}>t\right) \\
& =\int_{t}^{\infty} \int_{\arcsin \sqrt{t(1+s) / s(1+t)}}^{\pi / 2} \sin \theta \mathrm{d} \theta F_{S}(\mathrm{~d} s) \\
& =\frac{1}{\sqrt{1+t}} \int_{t}^{\infty} \sqrt{\frac{s-t}{s}} \mathrm{~d} F_{S}(s), \quad t \geq 0,
\end{aligned}
$$

for prolate case and

$$
\begin{aligned}
1-F_{T}(t) & =\mathbb{P}\left(S \sin ^{2} \Theta>t\right) \\
& =\int_{t}^{\infty} \int_{0}^{\arcsin \sqrt{t / s}} \sin \theta \mathrm{d} \theta F_{S}(\mathrm{~d} s) \\
& =\int_{t}^{\infty} \sqrt{\frac{s-t}{s}} \mathrm{~d} F_{S}(s), \quad t \geq 0,
\end{aligned}
$$

for oblate case, where $F_{S}$ is the distribution function of $S$. It will turn out to be useful to rewrite these formulas using integration by parts. For prolate spheroid it yields

$$
1-F_{T}(t)=\frac{1}{\sqrt{1+t}}-\frac{t}{\sqrt{1+t}} \int_{t}^{\infty} \frac{F_{S}(s)}{2 s^{3 / 2} \sqrt{s-t}} \mathrm{~d} s,
$$

which for $t>0$ becomes

$$
1-F_{T}(t)=\frac{t}{\sqrt{1+t}} \int_{t}^{\infty} \frac{1-F_{S}(s)}{2 s^{3 / 2} \sqrt{s-t}} \mathrm{~d} s, \quad t>0 .
$$

For oblate spheroid we end up with the following formula

$$
1-F_{T}(t)=t \int_{t}^{\infty} \frac{1-F_{S}(s)}{2 s^{3 / 2} \sqrt{s-t}} \mathrm{~d} s, \quad t>0 .
$$

We deal with the stereological unfolding problem. The estimation of unknown particle shape distribution function $F_{S}$ based on the estimator of $F_{T}$ is an ill-posed problem. Based on the extreme value theory we are going to study the tail behaviour of shape factor.

It is natural to use the semiaxes lengths as the size parameters. Since one semiaxis can be recovered from central section, we condition on the knowledge of its length and we are interested in the other semiaxis length, that is, $A$ for prolate spheroids, $C$ for oblate spheroids and $D$ for profiles. Similarly as for the shape factors, we can derive the relation between the conditional distributions of particle size parameter and profile size parameter $D$. For prolate spheroids, we have from Eq. 1,

$$
\begin{aligned}
1-F_{D \mid C}(d \mid c)=\mathbb{P}(D>d \mid C=c) \\
\quad=\mathbb{P}\left(\sin ^{2} \Theta>\frac{A^{2}\left(d^{2}-c^{2}\right)}{d^{2}\left(A^{2}-c^{2}\right)}\right) \\
\quad=\int_{d}^{\infty} \int_{\arcsin \sqrt{\frac{a^{2}\left(d^{2}-c^{2}\right)}{d^{2}\left(a^{2}-c^{2}\right)}} \sin \theta \mathrm{d} \theta F_{A \mid C}(\mathrm{~d} a \mid c)}^{\pi / 2} F^{\infty} \sqrt{\frac{a^{2}-d^{2}}{a^{2}-c^{2}}} F_{A \mid C}(\mathrm{~d} a \mid c), \quad d \geq c .
\end{aligned}
$$

We apply the integration by parts and obtain

$$
\begin{aligned}
& 1- F_{D \mid C}(d \mid c) \\
&= \frac{c\left(d^{2}-c^{2}\right)}{d} \int_{d}^{\infty} \frac{a\left(1-F_{A \mid C}(a \mid c)\right)}{\left(a^{2}-c^{2}\right)^{3 / 2} \sqrt{a^{2}-d^{2}}} \mathrm{~d} a, \\
& d>c .
\end{aligned}
$$

Clearly, $F_{D \mid C}(d \mid c)=0$ if $d<c$. For oblate spheroids we have

$$
\begin{aligned}
F_{D \mid A}(d \mid a) & =\mathbb{P}(D \leq d \mid A=a) \\
& =\mathbb{P}\left(\sin ^{2} \Theta \geq \frac{C^{2}\left(a^{2}-d^{2}\right)}{d^{2}\left(a^{2}-C^{2}\right)}\right) \\
& =\int_{0}^{d} \int_{\arcsin \sqrt{\frac{\left(a^{2}-d^{2}\right) c^{2}}{d^{2}\left(a^{2}-c^{2}\right)}}}^{\pi / 2} \theta \mathrm{d} \theta F_{C \mid A}(\mathrm{~d} c \mid a) \\
& =\int_{0}^{d} \frac{a}{d} \sqrt{\frac{d^{2}-c^{2}}{a^{2}-c^{2}}} F_{C \mid A}(\mathrm{~d} c \mid a), \quad d \leq a .
\end{aligned}
$$


If $F_{C \mid A}(0 \mid a)=0$, then the integration by parts implies

$$
\begin{aligned}
& F_{D \mid A}(d \mid a) \\
& =\frac{a\left(a^{2}-d^{2}\right)}{d} \int_{0}^{d} \frac{c F_{C \mid A}(c \mid a)}{\sqrt{d^{2}-c^{2}}\left(a^{2}-c^{2}\right)^{3 / 2}} \mathrm{~d} c, \\
& d<a .
\end{aligned}
$$

Clearly, $F_{D \mid A}(d \mid a)=1$ if $d>a$.

\section{EXTREME VALUE THEORY}

Let $Z_{1}, \ldots, Z_{n}$ be independent identically distributed random variables with common distribution function $F$. We are interested in the behaviour of the sample maximum $M_{n}=\max \left(Z_{1}, \ldots, Z_{n}\right)$. We say that $F$ belongs to the maximum domain of attraction of a distribution function $G$ if there exist normalizing constants $c_{n}>0$ and $d_{n} \in \mathbb{R}$ such that for all $z \in \mathbb{R}$ :

$$
\lim _{n \rightarrow \infty} F\left(c_{n} z+d_{n}\right)^{n}=G(z) .
$$

Since $\mathbb{P}\left(\frac{M_{n}-d_{n}}{c_{n}} \leq z\right)=\mathbb{P}\left(M_{n} \leq c_{n} z+d_{n}\right)=F\left(c_{n} z+\right.$ $\left.d_{n}\right)^{n}$, the limit relation Eq. 7 says that the distribution function of $\frac{M_{n}-d_{n}}{c_{n}}$ converges to the distribution function $G$. We shall write $F \in \operatorname{MDA}(G)$. The normalizing constants $c_{n}$ and $d_{n}$ are not given uniquely. For example, if we consider $c_{n}^{\prime}>0$ and $d_{n}^{\prime}$ such that

$$
\lim _{n \rightarrow \infty} \frac{c_{n}^{\prime}}{c_{n}}=1, \quad \lim _{n \rightarrow \infty} \frac{d_{n}^{\prime}-d_{n}}{c_{n}}=0,
$$

then Eq. 7 holds with $c_{n}$ replaced by $c_{n}^{\prime}$ and $d_{n}$ replaced by $d_{n}^{\prime}$. It is well-known that there are three possible non-degenerate limit distributions (FisherTippett theorem, cf. Embrechts et al. (1997), Theorem 3.2.3), $G$ belongs to the type of one of the following distribution functions $(\gamma>0)$ :

- Fréchet: $G_{1, \gamma}(z)=\exp \left\{-z^{-\gamma}\right\}, z>0$,

- Weibull: $G_{2, \gamma}(z)=\exp \left\{-(-z)^{\gamma}\right\}, z \leq 0$,

- Gumbel: $G_{3}(z)=\exp \left\{-\mathrm{e}^{-z}\right\}, z \in \mathbb{R}$.

We summarize several results concerning the characterization of $\operatorname{MDA}(G)$, for more details we refer to de Haan (1975) or Embrechts et al. (1997), Section 3.3. Let $\omega_{F}=\sup \{z \in \mathbb{R}: F(z)<1\}$ be the right endpoint of $F$ and let $F^{\leftarrow}(u)=\inf \{z \in$ $\mathbb{R}: F(z) \geq u\}, u \in(0,1)$, be the quantile function. The distribution function $F$ belongs to the maximum domain of attraction of $G_{1, \gamma}$ if and only if $\omega_{F}=\infty$ and

$$
\lim _{u \rightarrow \infty} \frac{1-F(u z)}{1-F(u)}=z^{-\gamma}, \quad \text { for all } z>0 .
$$

The normalizing constants can be chosen such that $c_{n}=F^{\leftarrow}(1-1 / n)$ and $d_{n}=0$. The distribution function $F$ belongs to the maximum domain of attraction of $G_{2, \gamma}$ if and only if $\omega_{F}<\infty$ and

$$
\lim _{u \rightarrow 0+} \frac{1-F\left(\omega_{F}-u z\right)}{1-F\left(\omega_{F}-u\right)}=z^{\gamma}, \quad \text { for all } z>0 .
$$

The normalizing constants can be chosen such that $c_{n}=\omega_{F}-F^{\leftarrow}(1-1 / n)$ and $d_{n}=\omega_{F}$. The distribution function $F$ belongs to the maximum domain of attraction of $G_{3}$ if and only if there exists some positive function $b$ such that

$$
\lim _{u \rightarrow \omega_{F}-} \frac{1-F(u+z b(u))}{1-F(u)}=\mathrm{e}^{-z}, \quad \text { for all } z \in \mathbb{R} .
$$

The auxiliary function $b$ may be chosen to be differentiable on $\left(-\infty, \omega_{F}\right)$ such that $\lim _{u \rightarrow \omega_{F}-} b^{\prime}(u)=0, \lim _{u \rightarrow \infty} b(u) / u=0$ if $\omega_{F}=\infty$ and $\lim _{u \rightarrow \omega_{F}-} b(u) /\left(\omega_{F}-u\right)=0$ if $\omega_{F}<\infty$. The normalizing constants can be chosen such that $d_{n}=$ $F^{\leftarrow}(1-1 / n)$ and $c_{n}=b\left(d_{n}\right)$.

Analogous considerations can be carried out for sample minima. A given distribution function belongs to the minimum domain of attraction of one of three distributions (Fréchet, Weibull or Gumbel). We give the characterization of the minimum domain of attraction of Weibull distribution. A distribution function $F$ belongs to the minimum domain of attraction of $G_{2, \gamma}$ if and only if $\eta_{F}>-\infty$ and

$$
\lim _{u \rightarrow 0+} \frac{1-F\left(\eta_{F}+u z\right)}{1-F\left(\eta_{F}+u\right)}=z^{\gamma}, \quad \text { for all } z>0,
$$

where $\eta_{F}=\inf \{z \in \mathbb{R}: F(z)>0\}$ is the left endpoint of $F$.

\section{TAIL BEHAVIOUR OF SHAPE AND SIZE PARAMETERS}

In this section we show the relation between the maximum domains of attraction of the shape and size parameters of profiles and particles. In the proofs we will often use the following lemma. It is a generalization of Lemma 1.2.1 in de Haan (1975) and it can also be found in Kötzer and Molchanov (2006) as Lemma 2.4.

Lemma 1. Let $f(\cdot, \cdot)$ and $g(\cdot, \cdot)$ be positive functions such that both

$$
\int_{0}^{\omega} f(s, t) \mathrm{d} t \quad \text { and } \quad \int_{0}^{\omega} g(s, t) \mathrm{d} t
$$


are finite for some $\omega \in(0, \infty]$ and for $s \leq t<\omega$,

$$
\lim _{s \rightarrow \omega} \frac{f(s, t)}{g(s, t)}=c \quad \text { with } \quad c \in[0, \infty] .
$$

Then

$$
\lim _{s \rightarrow \omega} \frac{\int_{s}^{\omega} f(s, t) \mathrm{d} t}{\int_{s}^{\omega} g(s, t) \mathrm{d} t}=c .
$$

Now we are ready to prove the stability of MDA for shape factors of spheroids. We start with prolate case.

Theorem 1. Let $\Xi_{1}, \ldots, \Xi_{n}$ be independent and identically distributed prolate spheroids with isotropic orientation. Assume that the orientation is independent of semiaxes lengths and that the $\Xi_{i}$ are not spheres with positive probability. Then the following assertions hold.

- If $F_{S} \in \operatorname{MDA}\left(G_{1, \gamma}\right)$, then $F_{T} \in \operatorname{MDA}\left(G_{1, \gamma+1 / 2}\right)$.

- If $F_{S} \in \operatorname{MDA}\left(G_{2, \gamma}\right)$, then $F_{T} \in \operatorname{MDA}\left(G_{2, \gamma+1 / 2}\right)$.

- If $F_{S} \in \operatorname{MDA}\left(G_{3}\right)$, then $F_{T} \in \operatorname{MDA}\left(G_{3}\right)$.

Proof. From Eq. 2 we observe that the right endpoints of $F_{T}$ and $F_{S}$ coincide, we denote them by $\omega$. Since we excluded the case $S=0$ a.s., we have $\omega>0$.

First we consider the maximum domain of attraction of Fréchet distribution. Applying Eq. 3 and Lemma 1 we find that

$$
\begin{aligned}
\lim _{u \rightarrow \infty} \frac{1-F_{T}(u z)}{1-F_{T}(u)} & =\lim _{u \rightarrow \infty} \frac{\frac{u z}{\sqrt{1+u z}} \int_{u z}^{\infty} \frac{1-F_{S}(s)}{2 s^{3 / 2} \sqrt{s-u z}} \mathrm{~d} s}{\frac{u}{\sqrt{1+u}} \int_{u}^{\infty} \frac{1-F_{S}(s)}{2 s^{3 / 2} \sqrt{s-u}} \mathrm{~d} s} \\
& =\lim _{u \rightarrow \infty} \frac{z \sqrt{1+u}}{\sqrt{1+u z}} \cdot \frac{\int_{u}^{\infty} \frac{1-F_{S}(v z)}{z^{2} v^{3 / 2} \sqrt{v-u}} z \mathrm{~d} v}{\int_{u}^{\infty} \frac{1-F_{S}(v)}{v^{3 / 2} \sqrt{v-u}} \mathrm{~d} v} \\
& =\lim _{u \rightarrow \infty} \frac{\sqrt{1+u}}{\sqrt{1+u z}} \cdot \frac{1-F_{S}(u z)}{1-F_{S}(u)} \\
& =z^{-1 / 2} \cdot z^{-\gamma}=z^{-(\gamma+1 / 2)}
\end{aligned}
$$

The case of MDA of Weibull distribution can be treated in a similar way:

$$
\begin{aligned}
\lim _{u \rightarrow 0+} & \frac{1-F_{T}(\omega-u z)}{1-F_{T}(\omega-u)} \\
= & \lim _{u \rightarrow 0+} \frac{\frac{\omega-u z}{\sqrt{1+\omega-u z}} \int_{\omega-u z}^{\omega} \frac{1-F_{S}(s)}{\frac{\omega-u}{2 s^{3 / 2} \sqrt{s-\omega+u z}}} \int_{\omega-u}^{\omega} \frac{1-F_{S}(s)}{2 s^{3 / 2} \sqrt{s-\omega+u}} \mathrm{~d} s}{\sqrt{1+\omega-u}} \\
= & \lim _{u \rightarrow 0+} \frac{(\omega-u z) \sqrt{1+\omega-u}}{(\omega-u) \sqrt{1+\omega-u z}} \cdot \frac{\int_{0}^{u} \frac{1-F_{S}(\omega-v z)}{(\omega-v z)^{3 / 2} \sqrt{(u-v) z}} z \mathrm{~d} v}{\int_{0}^{u} \frac{1-F_{S}(\omega-v)}{(\omega-v)^{3 / 2} \sqrt{u-v}} \mathrm{~d} v} \\
= & \sqrt{z} \lim _{u \rightarrow 0+} \frac{(\omega-u z) \sqrt{1+\omega-u}}{(\omega-u) \sqrt{1+\omega-u z}} \cdot \frac{1-F_{S}(\omega-u z)}{1-F_{S}(\omega-u)} \\
= & z^{\gamma+1 / 2} .
\end{aligned}
$$

Finally, we consider a Gumbel limiting distribution. Let $b$ be the auxiliary function from Eq. 8. Using the same arguments as in Drees and Reiss (1992), p. 211, we deduce that for some $u_{0} \in(0, \omega)$, the function $g: v \mapsto v+z b(v)$ is strictly increasing on $\left[u_{0}, \omega\right)$. Substituting $s=v+z b(v)$ we get

$$
\begin{aligned}
\lim _{u \rightarrow \omega-} & \frac{1-F_{T}(u+z b(u))}{1-F_{T}(u)} \\
= & \lim _{u \rightarrow \omega-} \frac{\frac{u+z b(u)}{\sqrt{1+u+z b(u)}} \int_{u+z b(u)}^{\omega} \frac{1-F_{S}(s)}{2 s^{3 / 2} \sqrt{s-u-z b(u)}} \mathrm{d} s}{\frac{u}{\sqrt{1+u}} \int_{u}^{\omega} \frac{1-F_{S}(s)}{2 s^{3 / 2} \sqrt{s-u}} \mathrm{~d} s} \\
= & \lim _{u \rightarrow \omega-} \frac{(u+z b(u)) \sqrt{1+u}}{u \sqrt{1+u+z b(u)}} \\
& \cdot \frac{\int_{u}^{g^{-1}}(\omega) \frac{1-F_{S}(v+z b(v))}{(v+z b(v))^{3 / 2} \sqrt{v+z b(v)-u-z b(u)}}\left(1+z b^{\prime}(v)\right) \mathrm{d} v}{\int_{u}^{\omega} \frac{1-F_{S}(v)}{v^{3 / 2} \sqrt{v-u}} \mathrm{~d} v} .
\end{aligned}
$$

The properties of $b$ and Eq. 8 imply $\lim _{u \rightarrow \omega-} \frac{(u+z b(u)) \sqrt{1+u}}{u \sqrt{1+u+z b(u)}}=1, \lim _{u \rightarrow \omega-}\left(1+z b^{\prime}(u)\right)=1$, $\lim _{u \rightarrow \omega-} \frac{u^{3 / 2}}{(u+z b(u))^{3 / 2}}=1$ and $\lim _{u \rightarrow \omega-} \frac{1-F_{S}(u+z b(u))}{1-F_{S}(u)}=$ $\mathrm{e}^{-z}$. Applying the mean value theorem and Lemma 1, we conclude with $\lim _{u \rightarrow \omega-} \frac{1-F_{T}(u+z b(u))}{1-F_{T}(u)}=\mathrm{e}^{-z}$.

It means that the distribution function of profile shapes belongs to the same maximum domain of attraction as the distribution function of particle shapes, only the parameter $\gamma$ may differ. Similar result holds for the population of oblate spheroids.

Theorem 2. Let $\Xi_{1}, \ldots, \Xi_{n}$ be independent and identically distributed oblate spheroids with isotropic orientation. Assume that the orientation is independent of semiaxes lengths and that the $\Xi_{i}$ are not spheres with positive probability. Then the following assertions hold.

- If $F_{S} \in \operatorname{MDA}\left(G_{1, \gamma}\right)$, then $F_{T} \in \operatorname{MDA}\left(G_{1, \gamma}\right)$.

- If $F_{S} \in \operatorname{MDA}\left(G_{2, \gamma}\right)$, then $F_{T} \in \operatorname{MDA}\left(G_{2, \gamma+1 / 2}\right)$.

- If $F_{S} \in \operatorname{MDA}\left(G_{3}\right)$, then $F_{T} \in \operatorname{MDA}\left(G_{3}\right)$.

Proof. The proof is analogous to that of Theorem 1. We only have to use Eq. 4 instead of Eq. 3. 
In the remainder of this section we are interested in the relation of tail distributions of size parameters of profiles and spheroids. We use the lengths of semiaxes as the size parameters. Recall that one semiaxis is always recovered from central section. Therefore, we condition on the knowledge of its length in our considerations. For the population of prolate spheroids we observe minor semiaxis of length $C$. Since $D \leq A$, large major semiaxis of the section profile can only be observed if the corresponding spheroid major semiaxis is large. Similarly as for the shape parameters, it turns out that the stability of the maximum domain of attraction follows.

Theorem 3. Let $\Xi_{1}, \ldots, \Xi_{n}$ be independent and identically distributed prolate spheroids with isotropic orientation. Assume that the orientation is independent of semiaxes lengths $A$ and $C$ and that the $\Xi_{i}$ are not spheres with positive probability. Then the following assertions hold.

- If $F_{A \mid C} \in \operatorname{MDA}\left(G_{1, \gamma}\right)$, then $F_{D \mid C} \in \operatorname{MDA}\left(G_{1, \gamma+1}\right)$.

- If $F_{A \mid C} \in \operatorname{MDA}\left(G_{2, \gamma}\right)$, then $F_{D \mid C} \in \operatorname{MDA}\left(G_{2, \gamma+1 / 2}\right)$.

- If $F_{A \mid C} \in \operatorname{MDA}\left(G_{3}\right)$, then $F_{D \mid C} \in \operatorname{MDA}\left(G_{3}\right)$.

Proof. The proof proceeds along the same lines as that of Theorem 1, except that we use Eq. 5. We just show how this works for the maximum domain of attraction of Fréchet distribution:

$$
\begin{aligned}
& \lim _{u \rightarrow \infty} \frac{1-F_{D \mid C}(u z \mid c)}{1-F_{D \mid C}(u \mid c)} \\
& =\lim _{u \rightarrow \infty} \frac{\frac{c\left(u^{2} z^{2}-c^{2}\right)}{u z} \int_{u z}^{\infty} \frac{a\left(1-F_{A \mid C}(a \mid c)\right)}{\left(a^{2}-c^{2}\right)^{3 / 2} \sqrt{a^{2}-u^{2} z^{2}}} \mathrm{~d} a}{\frac{c\left(u^{2}-c^{2}\right)}{u} \int_{u}^{\infty} \frac{a\left(1-F_{A \mid C}(a \mid c)\right)}{\left(a^{2}-c^{2}\right)^{3 / 2} \sqrt{a^{2}-u^{2}}} \mathrm{~d} a} \\
& =\lim _{u \rightarrow \infty} \frac{u^{2} z^{2}-c^{2}}{z\left(u^{2}-c^{2}\right)} \cdot \frac{\int_{u}^{\infty} \frac{v z\left(1-F_{A \mid C}(v z \mid c a)\right)}{\left(v^{2} z^{2}-c^{2}\right)^{3 / 2} z \sqrt{v^{2}-u^{2}}} z \mathrm{~d} v}{\int_{u}^{\infty} \frac{v\left(1-F_{A \mid C}(v \mid c)\right)}{\left(v^{2}-c^{2}\right)^{3 / 2} \sqrt{v^{2}-u^{2}}} \mathrm{~d} v} \\
& =z^{-1} \lim _{u \rightarrow \infty} \frac{1-F_{A \mid C}(u z \mid c)}{1-F_{A \mid C}(u \mid c)} \\
& =z^{-(\gamma+1)} \text {. }
\end{aligned}
$$

When considering oblate spheroids, the situation is reversed compared to the prolate case. We observe major semiaxis directly and minor semiaxis of the section profile is larger than minor semiaxis of the spheroid. It means that large minor semiaxis can be observed even if spheroid minor semiaxis is small. On the other hand, small minor semiaxis can only be observed if the corresponding spheroid minor semiaxis is small. Hence, we may investigate the lower tail behaviour of size parameters. Since the sizes are positive, we don't have to care about the minimum domain of attraction of Fréchet distribution. The following result states the stability property of the minimum domain of attraction of Weibull distribution.

Theorem 4. Let $\Xi_{1}, \ldots, \Xi_{n}$ be independent and identically distributed oblate spheroids with isotropic orientation. Assume that the orientation is independent of semiaxes lengths $A$ and $C$. Let the conditional distribution function $F_{C \mid A}$ of $C$ given $A$ have the left endpoint $\eta_{F}=0$ and $F_{C \mid A}(0 \mid a)=0$. If $F_{C \mid A}$ belongs to the minimum domain of attraction of $G_{2, \gamma}$, then $F_{D \mid A}$ belongs to the minimum domain of attraction of $G_{2, \gamma}$ as well.

Proof. Using a characterization of the minimum domain of attraction we get

$$
\lim _{u \rightarrow 0+} \frac{F_{C \mid A}(u z \mid a)}{F_{C \mid A}(u \mid a)}=z^{\gamma}
$$

for any $z>0$. Applying Eq. 6 we can write

$$
\begin{aligned}
\lim _{u \rightarrow 0+} \frac{F_{D \mid A}(u z \mid a)}{F_{D \mid A}(u \mid a)} \\
=\lim _{u \rightarrow 0+} \frac{\int_{0}^{u z} \frac{a c\left(a^{2}-u^{2} z^{2}\right)}{u z \sqrt{u^{2} z^{2}-c^{2}}\left(a^{2}-c^{2}\right)^{3 / 2}} F_{C \mid A}(c \mid a) \mathrm{d} c}{\int_{0}^{u} \frac{a c\left(a^{2}-u^{2}\right)}{u \sqrt{u^{2}-c^{2}}\left(a^{2}-c^{2}\right)^{3 / 2}} F_{C \mid A}(c \mid a) \mathrm{d} c} \\
=\lim _{u \rightarrow 0+} \frac{\int_{0}^{u} \frac{v z\left(a^{2}-u^{2} z^{2}\right)}{z^{2} \sqrt{u^{2}-v^{2}}\left(a^{2}-v^{2} z^{2}\right)^{3 / 2}} F_{C \mid A}(v z \mid a) z \mathrm{~d} v}{\int_{0}^{u} \frac{v\left(a^{2}-u^{2}\right)}{\sqrt{u^{2}-v^{2}}\left(a^{2}-v^{2}\right)^{3 / 2}} F_{C \mid A}(v \mid a) \mathrm{d} v} \\
=z^{\gamma},
\end{aligned}
$$

which completes the proof.

We also consider the maximum domain of attraction of Weibull distribution.

Theorem 5. Let $\Xi_{1}, \ldots, \Xi_{n}$ be independent and identically distributed oblate spheroids with isotropic orientation. Assume that the orientation is independent of semiaxes lengths $A$ and $C$. Let $F_{C \mid A}(0 \mid a)=0$. If $F_{C \mid A}$ belongs to the maximum domain of attraction of $G_{2, \gamma}$ with $\gamma>1$, then $F_{D \mid A}$ belongs to the maximum domain of attraction of $G_{2,1}$.

Proof. We have to show that

$$
\lim _{u \rightarrow 0+} \frac{1-F_{D \mid A}(a-u d \mid a)}{1-F_{D \mid A}(a-u \mid a)}=d .
$$


From Eq. 6, we get

$$
\begin{aligned}
& 1-F_{D \mid A}(a-u \mid a)= \\
& \quad u \frac{a(2 a-u)}{a-u} \int_{0}^{a-u} \frac{c\left(1-F_{C \mid A}(c \mid a)\right)}{\sqrt{(a-u)^{2}-c^{2}}\left(a^{2}-c^{2}\right)^{3 / 2}} \mathrm{~d} c .
\end{aligned}
$$

The assumption $F_{C \mid A} \in \operatorname{MDA}\left(G_{2, \gamma}\right)$ ensures that $1-$ $F_{C \mid A}(c \mid a)=(a-c)^{\gamma} L(a-c)$ for some slowly varying function $L$, i.e., $\lim _{u \rightarrow 0+} L(u z) / L(u)=1$ for any $z>$ 0 . Hence, we may apply the dominated convergence theorem and deduce that

$$
\begin{aligned}
& \lim _{u \rightarrow 0+} \frac{1-F_{D \mid A}(a-u \mid a)}{u} \\
&=2 a \int_{0}^{a} \frac{c\left(1-F_{C \mid A}(c \mid a)\right)}{\left(a^{2}-c^{2}\right)^{2}} \mathrm{~d} c<\infty .
\end{aligned}
$$

Therefore, we can conclude that

$$
\begin{aligned}
& \lim _{u \rightarrow 0+} \frac{1-F_{D \mid A}(a-u d \mid a)}{1-F_{D \mid A}(a-u \mid a)} \\
& \quad=d \lim _{u \rightarrow 0+} \frac{1-F_{D \mid A}(a-u d \mid a)}{u d} \frac{u}{1-F_{D \mid A}(a-u \mid a)} \\
& \quad=d .
\end{aligned}
$$

\section{WORKED EXAMPLE}

In this section we demonstrate the utility of our theoretical results on the following example. We consider a population of oblate spheroids and assume that the shape factor $S$ has distribution function $F_{S}$ with finite right endpoint $0<\omega<\infty$ and power law behaviour at $\omega$. Specifically,

$$
1-F_{S}(s)=K(\omega-s)^{\alpha}, \quad 0 \leq \omega-K^{-1 / \gamma} \leq s \leq \omega,
$$

for some $K, \gamma>0$. This ensures that $F_{S}$ belongs to the Weibull maximum domain of attraction $\operatorname{MDA}\left(G_{2, \gamma}\right)$. By Theorem 2, distribution function $F_{T}$ of profile shape factor $T$ belongs to $\operatorname{MDA}\left(G_{2, \gamma+1 / 2}\right)$. Using Eq. 4 we show that $F_{T}$ is tail-equivalent to a distribution function with power law behaviour at $\omega$.

Lemma 2. Let $F_{S}$ satisfy Eq. 9 and $F_{T}$ be given by Eq. 4, then

$$
\lim _{t \rightarrow \omega-} \frac{1-F_{T}(t)}{(\omega-t)^{\gamma+1 / 2}}=\frac{K}{2 \sqrt{\omega}} B\left(\gamma+1, \frac{1}{2}\right),
$$

where $B(\cdot, \cdot)$ is the beta function.
Proof. First we rewrite the complementary distribution function of $F_{T}$ using substitution $y=$ $(\omega-s) /(\omega-t)$,

$$
\begin{aligned}
\frac{1-F_{T}(t)}{(\omega-t)^{\gamma+1 / 2}} & =\int_{t}^{\omega} \frac{t K(\omega-s)^{\gamma}}{2 s^{3 / 2} \sqrt{s-t}(\omega-t)^{\gamma+1 / 2}} \mathrm{~d} s \\
& =\int_{0}^{1} \frac{t K y^{\gamma}(1-y)^{-1 / 2}}{2(\omega-(\omega-t) y)^{3 / 2}} \mathrm{~d} y .
\end{aligned}
$$

The statement of the lemma now follows by noting that

$$
\lim _{t \rightarrow \omega-} \frac{t}{(\omega-(\omega-t) y)^{3 / 2}}=\frac{1}{\sqrt{\omega}}
$$

and

$$
\int_{0}^{1} y^{\gamma}(1-y)^{-1 / 2} \mathrm{~d} y=B(\gamma+1,1 / 2) .
$$

Recall that the normalizing constants for $F_{S}$ can be chosen such that $c_{n}=\omega-F_{S}^{\leftarrow}(1-1 / n)$ and $d_{n}=\omega$. By Eq. 9, we get $c_{n}=(n K)^{-1 / \gamma}$, see also Embrechts et al. (1997), Example 3.3.16. Lemma 2 yields that the possible choice of normalizing constants for $F_{T}$ is $\tilde{c}_{n}=(n \tilde{K})^{-1 / \tilde{\gamma}}$ and $\tilde{d}_{n}=\omega$, where $\tilde{\gamma}=\gamma+$ $1 / 2$ and $\tilde{K}=\frac{K}{2 \sqrt{\omega}} B(\tilde{\gamma}+1 / 2,1 / 2)$. Since we want to estimate $c_{n}$ from estimate of $\tilde{c}_{n}$, the following relation turns out to be useful,

$$
c_{n}=\left(\tilde{c}_{n}^{\gamma+1 / 2} \frac{B\left(\gamma+1, \frac{1}{2}\right)}{2 \sqrt{\omega}}\right)^{\frac{1}{\gamma}} .
$$

In what follows, we consider a particular example that leads to the shape factor with power law behaviour at the right endpoint. A typical particle $\Xi_{0}$ is assumed to be oblate spheroid with semiaxes lengths $A$ and $C$ having joint density function

$$
f_{A, C}(a, c)= \begin{cases}\frac{8}{9}\left(\frac{a}{c}\right)^{3}, & \text { for } 1 \leq c \leq a \leq 2, \\ 0, & \text { otherwise } .\end{cases}
$$

After straightforward calculation one obtains distribution function $F_{S}$ of the shape factor $S=$ $A^{2} / C^{2}-1$,

$$
F_{S}(s)=1-\frac{1}{9}(3-s)^{2}, \quad 0 \leq s \leq 3 .
$$

We see that it is of the form Eq. 9 with $\gamma=2, K=$ $1 / 9$ and $\omega=3$. It is also possible to express $F_{T}$ by 
evaluating integral in Eq. 4,

$$
\begin{aligned}
& F_{T}(t)=1-\frac{\sqrt{3-t}}{\sqrt{3}}-\frac{\sqrt{3}}{18} t \sqrt{3-t} \\
&+\frac{t(12-t)}{18} \log \frac{\sqrt{3-t}+\sqrt{3}}{\sqrt{t}}, \\
& \quad 0<t \leq 3 .
\end{aligned}
$$

We generate a sample of $n=250$ independent isotropic oblate spheroids with semiaxes lengths distributed according to Eq. 11. For each spheroid a local section through its centre is performed, resulting in the collection of ellipses (profiles). Let $S_{1}, \ldots, S_{n}$ be the shape factors of simulated particles and let $T_{1}, \ldots, T_{n}$ be the shape factors of their profiles. Fig. 2 shows the histogram of particle shape factors together with theoretical density function. Of course, both are not available when observing only sectional data. On the other hand, Fig. 3 shows the histogram of shape factors obtained from central sections, this is the information that we have in practice when dealing with real data. For comparison, the theoretical density function (in practice unknown) of profile shapes is also depicted.

\section{Particle shapes}

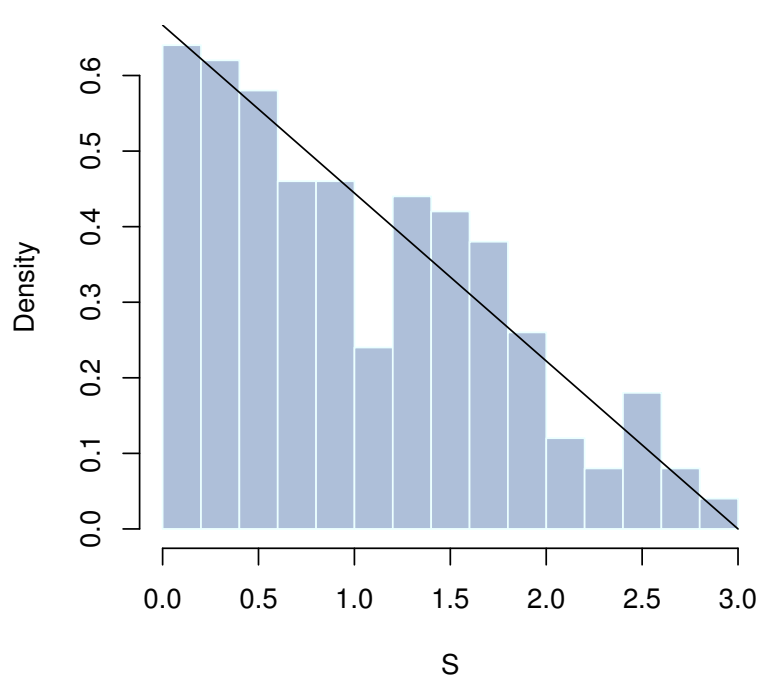

Fig. 2. Histogram of particle shape factors in simulated population of oblate spheroids together with theoretical density function corresponding to the distribution function given by Eq. 12.

\section{Profile shapes}

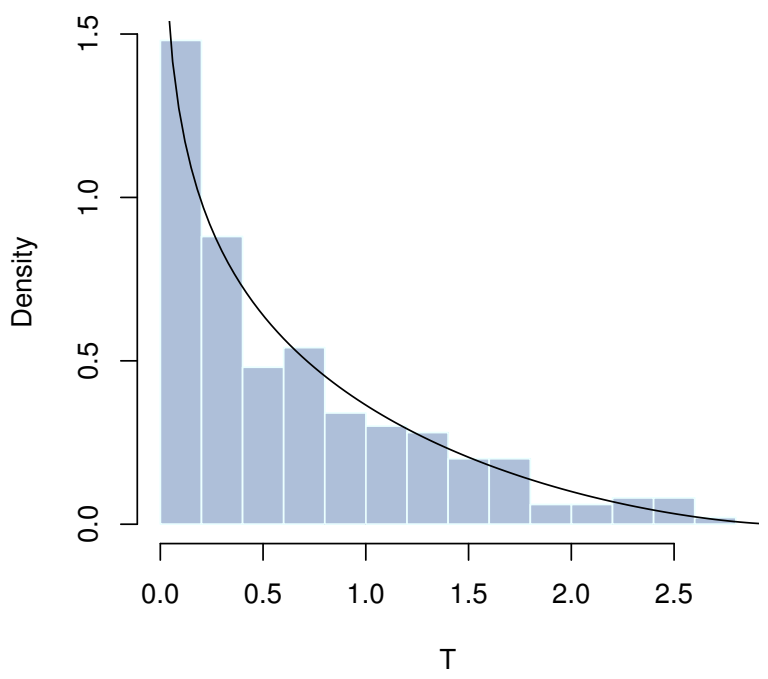

Fig. 3. Histogram of profile shape factors in simulated population of oblate spheroids together with theoretical density function derived from the distribution function given by Eq. 13.

Denote by $M_{n}=\max \left(S_{1}, \ldots, S_{n}\right)$ the maximal shape parameter. Our aim is to predict the distribution of $M_{n}$ based on observations of local section shapes $T_{1}, \ldots, T_{n}$. We order them from the largest to the smallest, i.e., $T_{(1)} \geq T_{(2)} \geq \cdots \geq T_{(n)}$ is the order statistics. We suppose that $F_{S} \in \operatorname{MDA}\left(G_{2, \gamma}\right)$ and that the right endpoint $\omega=3$ is known. By Theorem 2, $F_{T} \in \operatorname{MDA}\left(G_{2, \tilde{\gamma}}\right)$, where $\tilde{\gamma}=\gamma+1 / 2$. The index $\tilde{\gamma}$ can be estimated from the $k$ largest profile shape factors as

$$
\widehat{\tilde{\gamma}}=\left(\log \left(\omega-T_{(k)}\right)-\frac{1}{k} \sum_{i=1}^{k} \log \left(\omega-T_{(i)}\right)\right)^{-1},
$$

(Weissman, 1978). Then the estimate of $\gamma$ is $\hat{\gamma}=\widehat{\tilde{\gamma}}-\frac{1}{2}$. Furthermore, we estimate $\tilde{c}_{n}$ by

$$
\widehat{\tilde{c}}_{n}=n^{-\frac{1}{\widehat{\gamma}}} \omega \text {. }
$$

Finally, the estimator of $c_{n}$ is obtained by plugging the estimates $\hat{\gamma}$ and $\widehat{\tilde{c}}_{n}$ into Eq. 10:

$$
\hat{c}_{n}=\left(\hat{\tilde{c}}_{n}^{\hat{\gamma}+1 / 2} \frac{B\left(\hat{\gamma}+1, \frac{1}{2}\right)}{2 \sqrt{\omega}}\right)^{\frac{1}{\hat{\gamma}}} .
$$

The estimators depend on the choice of $k$. For our purposes, we take $k=10$.

The distribution function of $M_{n}$ is $F_{M_{n}}(s)=$ $\mathbb{P}\left(M_{n} \leq s\right)=F_{S}(s)^{n}$ and for large $n$ it can be approximated by $G_{2, \gamma}\left(\frac{s-d_{n}}{c_{n}}\right), c f$. Eq. 7. By replacing 
$\gamma$ and $c_{n}$ with their estimates, we obtain the asymptotic approximation of the distribution function of $M_{n}$ in the form

$$
\hat{F}_{M_{n}}(s)=G_{2, \hat{\gamma}}\left(\frac{s-\omega}{\hat{c}_{n}}\right), \quad s \leq \omega .
$$

The graph of this function is shown in Fig. 4. It is compared with true (unknown) distribution function of $M_{n}$.

\section{Distribution function of maximal particle shape}

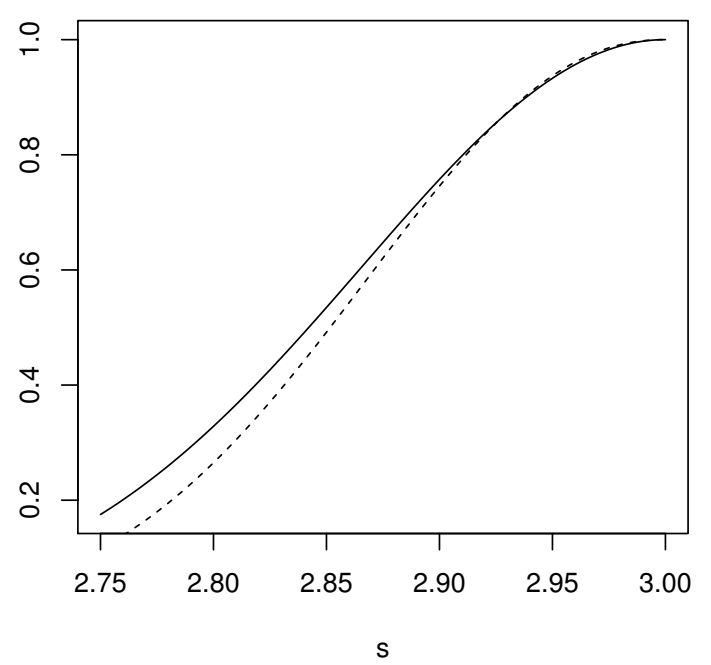

Fig. 4. The resulting asymptotic approximation (dashed line) of $F_{M_{n}}$ compared with true distribution function (solid line).

\section{CONCLUSION}

We proved stability properties of the domain of attraction for shape and size parameters of spheroidal particles under local stereological sampling design. They can be helpful for estimating stereologically the tail of a particle parameter distribution. Practical applications are illustrated by an example applied to the shape factor of oblate spheroids generated by computer simulations. Analogous applications for the case of isotropic uniform random probes are discussed in Hlubinka (2003b) or Hlubinka (2006b). First we choose a parametric model for spheroid parameter of interest. It belongs to the domain of attraction of some distribution function. Then our theoretical results say that profile parameter belongs to the same domain of attraction with possibly different parameter $\gamma$. From sectional data we find the estimates $\widehat{\widetilde{c}}_{n}$ and $\widehat{\tilde{d}}_{n}$ of normalizing constants $\tilde{c}_{n}$ and $\tilde{d}_{n}$ for the observed profile parameter. The normalizing constants $c_{n}$ and $d_{n}$ for distribution function of spheroid parameter are then estimated by $\hat{c}_{n}$ and $\hat{d}_{n}$ using the relations between $\left(c_{n}, d_{n}\right)$ and $\left(\tilde{c}_{n}, \tilde{d}_{n}\right)$ and estimators $\widehat{\tilde{c}}_{n}$ and $\widehat{\tilde{d}}_{n}$. Finally, the distribution of extremal spheroid parameter is approximated by the limiting distribution (Fréchet, Weibull or Gumbel) with normalizing constants $\hat{c}_{n}$ and $\hat{d}_{n}$.

\section{ACKNOWLEDGEMENTS}

The work is supported by the Grant Agency of the Czech Republic, project P201/10/0472. An earlier (non-reviewed) version of this paper was presented at the 13th International Congress for Stereology and appeared in the Congress proceedings (Pawlas, 2011).

\section{REFERENCES}

Beneš V, Rataj J (2004). Stochastic geometry: Selected topics. Boston: Kluwer Academic Publishers.

Bortot P, Coles SG, Sisson SA (2007). Inference for stereological extremes. J Am Stat Assoc 102:84-92.

de Haan L (1975). On regular variation and its application to the weak convergence of sample extremes, 3rd printing. Mathematical Centre Tracts 32, Mathematisch Centrum, Amsterdam.

Drees H, Reiss RD (1992). Tail behaviour in Wicksell's corpuscle problem. In: Galambos J, Kátai I, eds. Probability Theory and Applications - Essays to the Memory of József Megyoródi. Dordrecht: Kluwer Academic Publishers, 205-20.

Embrechts P, Klüppelberg C, Mikosch T (1997). Modelling extremal events. Berlin: Springer.

Hlubinka D (2003a). Stereology of extremes; shape factor of spheroids. Extremes 6:5-24.

Hlubinka D (2003b). Stereology of extremes; size of spheroids. Math Bohem 128:419-38.

Hlubinka D (2006a). Extremes of spheroid shape factor based on two dimensional profiles. Kybernetika 62:7794.

Hlubinka D (2006b). Shape factor extremes for prolate spheroids. Kybernetika 62:557-68.

Hlubinka D (2006c). Size and shape factor extremes of spheroids. Image Anal Stereol 25:145-54.

Hlubinka D, Kotz S (2010). The generalized FGM distribution and its application to stereology of extremes. Appl Math 55:495-512.

Kötzer S, Molchanov I (2006). On the domain of attraction for the lower tail in Wicksell's corpuscle problem. In: Lechnerová R, Saxl I, Beneš V, eds. Proc $\mathrm{S}^{4} \mathrm{G}$. Prague: Union of Czech Mathematicians and Physicists. 91-6.

Molchanov I (2005). Theory of random sets. London: Springer. 
Pawlas Z, Nyengaard JR, Vedel Jensen EB (2009). Particle sizes from sectional data. Biometrics 65:216-24.

Pawlas Z (2011). Local stereology of extremes. In: Guoquan Liu et al., eds. Proc 13th Int Congr Stereol. Beijing: Chinese Society for Stereology.

Takahashi R, Sibuya M (1996). The maximum size of the planar sections of random spheres and its application to metallurgy. Ann Inst Stat Math 48:127-44.

Takahashi R, Sibuya M (1998). Prediction of the maximum size in Wicksell's corpuscle problem. Ann Inst Stat Math 50:361-77.

Takahashi R, Sibuya M (2001). Prediction of the maximum size in Wicksell's corpuscle problem, II. Ann Inst Stat
Math 53:647-60.

Takahashi R, Sibuya M (2002). Metal fatigue, Wicksell transform and extreme values. Appl Stochast Models Bus Ind 18:301-12.

Vedel Jensen EB (1998). Local stereology. Singapore: World Scientific.

Weissman I (1978). Estimation of parameters and larger quantiles based on the $k$ largest observations. J Am Stat Assoc 73:812-5.

Wicksell SD (1925). The corpuscle problem. A mathematical study of a biometric problem. Biometrika 17:84-99. 